\title{
Erratum to: Proposed model for growth preference of plate-like nanohydroxyapatite crystals on superhydrophilic vertically aligned carbon nanotubes by electrodeposition
}

\author{
A. O. Lobo - F. R. Marciano - I. Regiani \\ S. C. Ramos · J. T. Matsushima $\cdot$ E. J. Corat
}

Published online: 4 November 2011

(C) Springer-Verlag 2011

\section{Erratum to: Theor Chem Acc \\ DOI 10.1007/s00214-011-0993-x}

The original publication of Table 3 in the article was published with errors in the values of the crystallites (column 6) and no units were included for the FWHM numbers (column 5). The units for the FWHM values and the correct crystallite values are given in the complete version below:

The online version of the original article can be found under doi:10.1007/s00214-011-0993-x.

A. O. Lobo $(\bowtie) \cdot$ F. R. Marciano

Laboratorio de Nanotecnologia Biomedica (NanoBio),

Universidade do Vale do Paraíba (UniVaP), Avenida Shishima

Hifumi 2911, Urbanova, São José dos Campos,

SP 12244-000, Brazil

e-mail: loboao@yahoo.com

\section{A. O. Lobo · F. R. Marciano}

Laboratorio de Espectroscopia Vibracional Biomedica (LEVB), Instituto de Pesquisa e Desenvolvimento, Universidade do Vale do Paraiba, Avenida Shishima Hifumi 2911, Urbanova,

São José dos Campos, SP 12244-000, Brazil

\section{Regiani}

Instituto Tecnológico de Aeronáutica (ITA), Praça Marechal Eduardo Gomes 50, Vila das Acácias, São José dos Campos, SP 12228-900, Brazil

S. C. Ramos · J. T. Matsushima · E. J. Corat Laboratorio Associado de Sensores e Materiais (LAS), Instituto Nacional de Pesquisas Espaciais (INPE), Avenida dos Astronautas 1758, Jardin da Granja, São José dos Campos, SP 12227-010, Brazil
Table 3 Parameters collected from deconvolution of the X-ray diffractogram of plate-like n-HA crystals electrodeposited on superhydrophilic VACNT films

\begin{tabular}{llllllc}
\hline Plane & $\begin{array}{l}\text { Position } \\
(2-t h e t a)\end{array}$ & $\begin{array}{l}\text { Intensity } \\
(\text { arb. } \\
\text { units })\end{array}$ & $\begin{array}{l}\text { Intensity } \\
(\text { JPCD })^{\mathrm{a}}\end{array}$ & $\begin{array}{l}\text { FWHM } \\
\left({ }^{\circ}\right)\end{array}$ & $\begin{array}{l}\text { Crystallites } \\
(\mathrm{nm})\end{array}$ & $\begin{array}{l}\text { Preference } \\
\text { growth, P }\end{array}$ \\
\hline$(002)$ & 25.9 & 0.45 & 0.4 & 0.46 & 18.95 & 1.5 \\
$(211)$ & 31.7 & 0.32 & 1.0 & 0.31 & 22.56 & -0.5 \\
$(112)$ & 32.2 & 0.34 & 0.6 & 0.50 & 18.51 & 0.1 \\
$(300)$ & 32.7 & 0.23 & 0.6 & 0.24 & 18.61 & -0.2 \\
\hline
\end{tabular}

All crystallite sizes and growth preferences determined for the planes are shown. The JPCDS 9-432 was used for comparison

a Data collected for HA standard powder (JPCD 9-432) for preference growth compute 